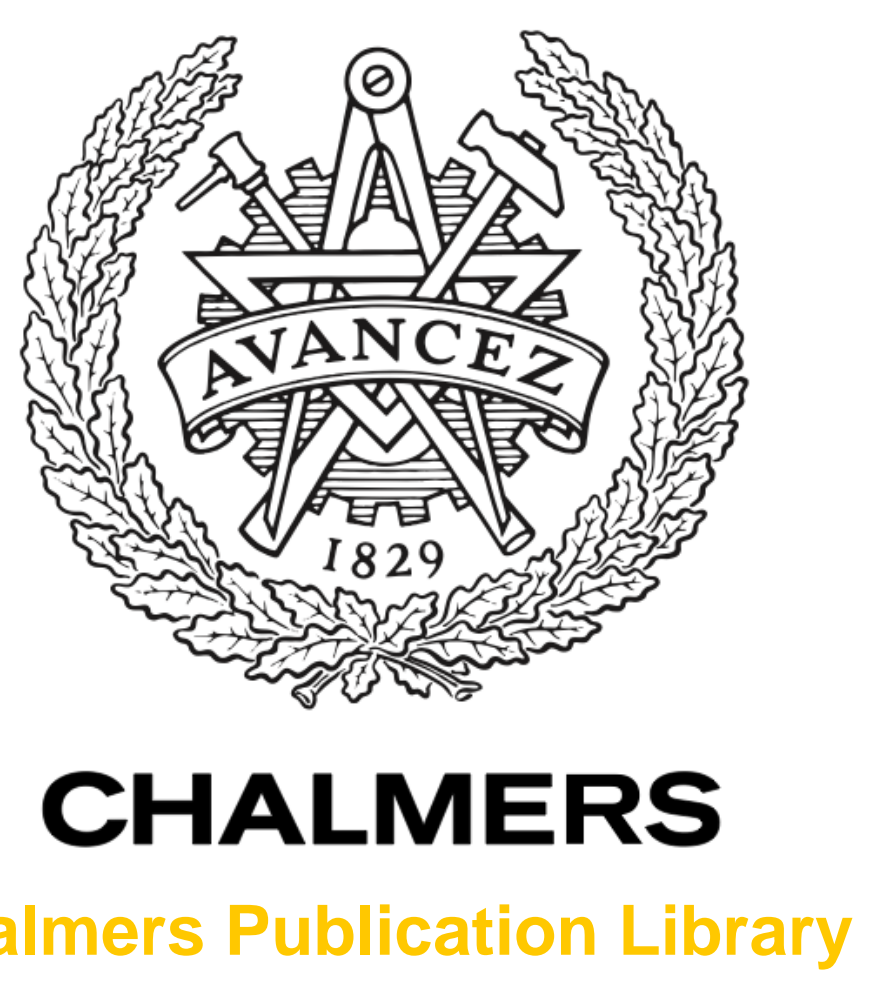

Chalmers Publication Library

Wireless Information and Power Transfer in MIMO Channels under Rician fading

This document has been downloaded from Chalmers Publication Library (CPL). It is the author's version of a work that was accepted for publication in:

Proc. 2015 IEEE International Conference on Acoustics, Speech and Signal Processing (ICASSP)

Citation for the published paper:

Ozcelikkale, A. ; McKelvey, T. ; Viberg, M. (2015) "Wireless Information and Power

Transfer in MIMO Channels under Rician fading". Proc. 2015 IEEE International

Conference on Acoustics, Speech and Signal Processing (ICASSP) pp. 3187-3191.

Downloaded from: http://publications.lib.chalmers.se/publication/230608

Notice: Changes introduced as a result of publishing processes such as copy-editing and formatting may not be reflected in this document. For a definitive version of this work, please refer to the published source. Please note that access to the published version might require a subscription. 


\title{
WIRELESS INFORMATION AND POWER TRANSFER IN MIMO CHANNELS UNDER RICIAN FADING
}

\author{
Ayça Özçelikkale, Tomas McKelvey, Mats Viberg
}

\author{
Dept. of Signals and Systems, Chalmers University of Technology, Gothenburg, Sweden
}

\begin{abstract}
Simultaneous wireless information and power transfer (SWIPT) offers an attractive alternative to the traditional battery limited or grid dependent communication system design. In this paper, we investigate robust precoder designs for systems with SWIPT capabilities under a stochastic Rician fading framework. Under a multiple-input multiple-output (MIMO) channel model, we formulate the problem of minimizing the average mean-square error at the information receiver (IR) while keeping the average energy harvested at the energy receiver (ER) above given levels. We consider two different strategies that can be adopted by the IR: i) simple estimation filters based solely on the channel mean, ii) robust estimation filters aiming to minimize the average mean-square error. Both of these scenarios lead to non-convex formulations. For the first scenario, we propose a convex relaxation that is tight. For the second scenario, we propose an alternating minimization method that provides precoder designs even in the scenarios where the number of transmit antennas is larger than the length of data vector. Our numerical results show that the proposed designs provide significant performance gains especially when the scattering component of the channel is strong.
\end{abstract}

Index Terms - wireless power transfer, robust precoder design, fading.

\section{INTRODUCTION}

Simultaneous wireless information and power transfer offers an attractive alternative to the traditional battery limited or grid dependent communication system design. SWIPT approach brings flexibility in terms of power management in a wide range of applications including wireless sensor networks and smart homes. In systems with SWIPT capabilities, the two tasks, information and power transfer is done simultaneously in a wireless medium. The optimal transmission strategies for these two tasks are different, hence novel transmission strategies have to be designed in order to be able to do these two tasks most efficiently [1-3].

A practical issue in communication system design for multiple antenna systems is the design of precoders and receiver filters. Previously precoder and receiver filter designs have been successfully used to improve communication systems performance for systems without energy transfer capabilities, see for instance [4-6]. Typically, joint linear precoder-receiver filter design requires the channel information to be available to system designers. On the other hand, in practice, the channel state information (CSI) cannot be obtained perfectly. Hence, investigation of the effect of partial CSI and development of robust designs is of paramount importance from a practical implementation point of view. In this regard, a number of works investigate the robust designs for SWIPT systems. Norm bounded deterministic channel estimation error models are investigated for multiple-input single-output (MISO) multiuser scenario [7], relay channels [8] and secure communication scenarios $[9,10]$. Robust designs in stochastic settings are investigated in $[11,12]$.
In this paper, we investigate robust precoder designs for simultaneous information and energy transfer under a stochastic framework, in particular Rician fading. We utilize mean-square error as the performance metric which is a widely used metric for precoder design, see for instance [4-6]. Under a MIMO channel model, we formulate the problem of minimizing the average mean-square error while keeping the average energy harvested at the energy receiver above a given level. We consider two different information receiver structures. For the case where the receiver filter is based on only the average channel information, we seek for the optimal linear precoders at the transmitter. For this non-convex problem, we propose a convex relaxation that is tight (Sec. 3). For the scenario where the information receiver and the transmitter jointly optimize the transmission strategy, i.e. linear precoder and the IR filter, we propose an alternating minimization method (Sec. 4). For this second case, our framework is similar to [11] where mean-square error criterion together with rate maximization is considered under a similar fading scenario. Nevertheless, unlike [11] our formulation lets us investigate the scenarios with receiver antenna correlation at the information receiver and the cases where the number of antennas at the transmitter is larger than the length of data vector. This allows us to offer designs that can take advantage of possibly large number of antennas at the transmitter, for instance as in massive MIMO applications.

The rest of the paper is organized as follows. In Sec. 2, the system model is described. The scenario where the receiver filter is fixed and is based on only the average channel information is discussed in Sec. 3. The joint precoder-receiver filter optimization problem is investigated in Sec. 4. In Sec. 5, performance of our robust designs are illustrated. Finally, the paper is concluded in Sec. 6.

The following notation is used throughout the paper. Uppercase and lowercase letters denote matrices, and column/row vectors, respectively. The complex conjugate transpose, transpose and pseudoinverse of a matrix $\mathbf{A}$ are denoted by $\mathbf{A}^{\mathrm{H}}, \mathbf{A}^{\mathrm{T}}$ and $\mathbf{A}^{+}$, respectively. The $i$ th row $j$ th column element of matrix $\mathbf{A}$ is denoted by $[\mathbf{A}]_{i j}$. The Frobenius norm of a matrix $\mathbf{A}$ is denoted by $\|\mathbf{A}\|^{2}=\operatorname{tr}\left[\mathbf{A} \mathbf{A}^{\mathrm{H}}\right]$. $\mathbf{I}$ denotes the identity matrix with the suitable dimensions. Positive semi-definite ordering is denoted by $\succeq$, where $\mathbf{A} \succeq 0$ denotes a Hermitian positive semi-definite matrix. An optimal value of an optimization variable $\mathbf{A}$ is denoted by $\mathbf{A}^{*}$. The operators $\mathrm{E}[$.$] , and \operatorname{tr}[$. denote the expectation and trace operators, respectively.

\section{SYSTEM MODEL}

\subsection{Channel and Signal Models}

The multi-antenna transmitter transfer information to the information receiver as well as power to the energy harvesting receiver according to

$$
\begin{aligned}
\mathbf{y}_{\mathbf{I}} & =\mathbf{H}_{\mathbf{I}} \mathbf{x}+\mathbf{w}_{\mathbf{I}} \\
\mathbf{y}_{\mathbf{E}} & =\mathbf{H}_{\mathbf{E}} \mathbf{x}+\mathbf{w}_{\mathbf{E}}
\end{aligned}
$$

where $\mathbf{H}_{\mathbf{I}} \in \mathbb{C}^{n_{r} \times n_{t}}$ and $\mathbf{H}_{\mathbf{E}} \in \mathbb{C}^{n_{e} \times n_{t}}$ represent the channel gains from the transmitter to the information receiver and the energy 
receiver, respectively. Zero-mean complex proper Gaussian random variables $\mathbf{w}_{\mathbf{I}} \in \mathbb{C}^{n_{r} \times 1} \sim \mathcal{C N}\left(\mathbf{0}, \mathbf{K}_{\mathbf{w}_{\mathbf{I}}}\right), \mathbf{K}_{\mathbf{w}_{\mathbf{I}}}=\mathrm{E}\left[\mathbf{w}_{\mathbf{I}} \mathbf{w}_{\mathbf{I}}^{\mathrm{H}}\right]$ and $\mathbf{w}_{\mathbf{E}} \in \mathbb{C}^{n_{e} \times 1} \sim \mathcal{C N}\left(\mathbf{0}, \mathbf{K}_{\mathbf{w}_{\mathbf{E}}}\right), \mathbf{K}_{\mathbf{w}_{\mathbf{E}}}=\mathrm{E}\left[\mathbf{w}_{\mathbf{E}} \mathbf{w}_{\mathbf{E}}^{\mathrm{H}}\right]$ denote the noise at the IR's and ER's channel, respectively.

The channel input $\mathbf{x}$ is formed as $\mathbf{x}=\mathbf{A s}$, where the zero mean complex proper Gaussian random vector $\mathbf{s} \in \mathbb{C}^{n_{s}}, \mathbf{s} \sim \mathcal{C N}\left(\mathbf{0}, \mathbf{K}_{\mathbf{s}}\right)$, $\mathbf{K}_{\mathbf{s}}=\mathbf{I}$ denotes the data and $\mathbf{A} \in \mathbb{C}^{n_{t} \times n_{s}}$ denotes the linear precoder. The following transmission power constraint is imposed at the transmitter

$$
\operatorname{tr}\left[\mathbf{A A}^{\mathrm{H}}\right] \leq P .
$$

We assume that both the IR and ER channels go under Rician fading. In particular, the channel for the information receiver can be expressed as follows

$$
\mathbf{H}_{\mathbf{I}}=\mathbf{H}_{\mathbf{I}}^{\mathbf{0}}+\mathbf{T}_{\mathbf{I}}
$$

where $\mathbf{H}_{\mathbf{I}}^{0}$ represents the deterministic line-of-sight component of the channel, and $\mathbf{T}_{\mathbf{I}}$ represents the scattering component with Ragleigh distribution. In particular, we have

$$
\mathbf{T}_{\mathbf{I}}=\mathbf{R}_{\mathbf{I R}}{ }^{1 / 2} \overline{\mathbf{T}}_{\mathbf{I}}\left(\mathbf{R}_{\mathbf{T}}{ }^{1 / 2}\right)^{\mathrm{T}}
$$

where elements of $\overline{\mathbf{T}}_{\mathbf{I}}$ are i.i.d. complex proper Gaussian variables with $\left[\overline{\mathbf{T}}_{\mathbf{I}}\right]_{i, j} \sim \mathcal{C N}\left(0, \sigma_{T, I}^{2}\right)$ [13-15]. Here the covariance matrices $\mathbf{R}_{\mathbf{T}} \succeq 0$ and $\mathbf{R}_{\mathbf{I R}} \succeq 0$ model the channel correlation at the transmitter side, and the channel correlation at the receiver side, respectively. The channel for the energy receiver is modelled similarly with $\mathbf{H}_{\mathbf{E}}^{\mathbf{0}}$, $\mathbf{T}_{\mathbf{E}}, \mathbf{R}_{\mathbf{E R}}$ defined in the same manner. We note that the transmitter side correlation is given by $\mathbf{R}_{\mathbf{T}}$ for both channels since the same transmitter serves both to the information and the energy receiver. It is assumed that $\overline{\mathbf{T}}_{\mathbf{I}}, \overline{\mathbf{T}}_{\mathbf{E}}, \mathbf{w}_{\mathbf{I}}, \mathbf{w}_{\mathbf{E}}$, and $\mathbf{s}$ are statistically independent.

\subsection{Signal Recovery at the IR}

Upon receiving $\mathbf{y}_{\mathbf{I}}$, the information receiver forms an estimate of $\mathbf{s}$. For a given realization $\mathbf{H}_{\mathbf{I}}$, the mean-square error is given by

$$
\varepsilon(\mathbf{A}, \mathbf{B})=\mathrm{E}_{S}\left[\left\|\mathbf{s}-\mathbf{B y}_{\mathbf{I}}\right\|^{2}\right]
$$

where $\mathbf{B}$ represents the linear estimator adopted by the receiver. The performance criterion at the information receiver is the average mean square error

$$
\mathrm{E}_{H}[\varepsilon(\mathbf{A}, \mathbf{B})]=\mathrm{E}_{S, H}\left[\left\|\mathbf{s}-\mathbf{B y}_{\mathbf{I}}\right\|^{2}\right]
$$

Here the subscripts $S, H$ for the expectation operator is used to denote the expectation with respect to signals and the channels respectively. We consider the following estimation strategies that can be adopted by the IR:

Scenario I - Channel Equalizer: IR uses an estimator B that only depends on the channel mean $\mathbf{H}_{\mathbf{I}}^{0}$ and is independent of the precoder design. Such models have been used in various partial CSI scenarios successfully, see for instance [16]. This model includes a wide range of channel equalizer scenarios, such as the zero forcing equalizer $\left(\mathbf{H}_{\mathbf{I}}^{0^{+}}\right)$, and the mean-square error equalizer $\left(\mathbf{H}_{\mathbf{I}}^{0^{\mathrm{H}}}\left(\mathbf{H}_{\mathbf{I}}^{0} \mathbf{H}_{\mathbf{I}}^{0^{\mathrm{H}}}+\right.\right.$ $\left.\mathbf{K}_{\mathbf{w}_{\mathbf{I}}}\right)^{-1}$ ). Since the receiver strategies do not depend on the transmitter strategy, such frameworks have the advantage of backward compatibility.

Scenario II -General Filtering: IR uses an estimator B that depends on $\mathbf{H}_{\mathbf{I}}^{\mathbf{0}}, \mathbf{A}$ and channel statistics. This case is more suitable for scenarios with more complicated receiver structures and where joint filter design for the transmitter and receiver is possible. In this case, for a given linear precoding strategy $\mathbf{A}$ at the transmitter, the receiver uses the following estimator $\mathbf{B}^{*}$ which minimizes the average error

$$
\mathbf{B}^{*}\left(\mathbf{H}_{\mathbf{I}}^{\mathbf{0}}, \mathbf{A}, \mathbf{R}_{\mathbf{T}}, \mathbf{R}_{\mathbf{I R}}\right)=\arg \min _{\mathbf{B}} \mathbf{E}_{S, H}\left[\left\|\mathbf{s}-\mathbf{B} \mathbf{y}_{\mathbf{I}}\right\|^{2}\right]
$$

This receiver filter design problem is further discussed in Sec. 4.

\subsection{Energy Harvesting at the ER}

For a given $\mathbf{H}_{\mathbf{E}}$, the energy harvested at the ER can be expressed as follows [1]

$$
\mathcal{J}(\mathbf{A})=\operatorname{tr}\left[\mathbf{H}_{\mathbf{E}} \mathbf{A} \mathbf{A}^{\mathrm{H}} \mathbf{H}_{\mathbf{E}}{ }^{\mathrm{H}}\right] .
$$

The following average energy harvesting constraint is imposed at the energy receiver:

$$
\mathrm{E}_{H}[\mathcal{J}(\mathbf{A})] \geq \gamma
$$

We note that it is possible to account for the possible loss in the energy conversion process by scaling right hand side expression in (7) with a loss factor $\kappa \in[0,1]$. Here, we assume that this loss is accounted for while setting desired energy levels.

\subsection{Precoder Design Problem}

For Scenario I, for a given receiver filter $\mathbf{B}\left(\mathbf{H}_{\mathbf{I}}^{0}\right)$, we are interested in the following precoder filter design problem

$(P 1)$

$$
\begin{array}{cl}
\min _{\mathbf{A}} & \left.\mathrm{E}_{H}\left[\varepsilon\left(\mathbf{A}, \mathbf{B}\left(\mathbf{H}_{\mathbf{I}}^{\mathbf{0}}\right)\right)\right)\right] \\
\text { s.t. } & \text { (3) and (8). }
\end{array}
$$

For Scenario II, we are interested in the following joint precoder and receiver filter design problem

$$
\begin{aligned}
(P 2) \quad \min _{\mathbf{A}, \mathbf{B}} & \mathrm{E}_{H}[\varepsilon(\mathbf{A}, \mathbf{B})] \\
\text { s.t. } & (3) \text { and (8). }
\end{aligned}
$$

In both scenarios we minimize the average mean-square error at the information receiver while satisfying the transmit power constraint at the transmitter and the energy harvesting requirement at the energy receiver. We discuss these design problems in more detail in Sec. 3 for Scenario I and in Sec. 4 for Scenario II.

\section{PRECODER DESIGN WITH FIXED RECEIVER FILTER AT THE IR}

In this section, we focus on Scenario I and discuss the precoder design problem in (9). We first present the explicit forms of the objective function and the constraints, and discuss the convexity properties of the problem. Although the problem is non-convex, we show that it is possible to solve it by convex optimization methods by using a tight convex relaxation.

For a given channel realization, the mean-square error at the IR can be written as

$$
\begin{aligned}
\varepsilon(\mathbf{A}, \mathbf{B}) & =\mathrm{E}_{S}\left[\left\|\mathbf{s}-\mathbf{B} \mathbf{y}_{\mathbf{I}}\right\|^{2}\right] \\
& =\mathrm{E}_{S}\left[\left\|\mathbf{s}-\mathbf{B}\left(\mathbf{H}_{\mathbf{I}} \mathbf{A} \mathbf{s}+\mathbf{w}_{\mathbf{I}}\right)\right\|^{2}\right] \\
& =\left\|\mathbf{I}-\mathbf{B} \mathbf{H}_{\mathbf{I}} \mathbf{A}\right\|^{2}+\operatorname{tr}\left[\mathbf{B} \mathbf{K}_{\mathbf{w}_{\mathbf{I}}} \mathbf{B}^{\mathrm{H}}\right]
\end{aligned}
$$

where for notational convenience we have expressed $\mathbf{B}\left(\mathbf{H}_{\mathbf{I}}^{\mathbf{0}}\right)$ as $\mathbf{B}$. Hence the average mean-square error can be expressed as $\mathrm{E}_{H}[\varepsilon(\mathbf{A}, \mathbf{B})]=\mathrm{E}_{H, S}\left[\left\|\mathbf{s}-\mathbf{B y}_{\mathbf{I}}\right\|^{2}\right]$

$$
\begin{aligned}
& =\mathrm{E}_{H}\left[\left\|\left(\mathbf{I}-\mathbf{B H}_{\mathbf{I}}^{\mathbf{0}} \mathbf{A}\right)-\mathbf{B} \mathbf{T}_{\mathbf{I}} \mathbf{A}\right\|^{2}\right]+\operatorname{tr}\left[\mathbf{B} \mathbf{K}_{\mathbf{w}_{\mathbf{I}}} \mathbf{B}^{\mathrm{H}}\right] \\
& =\left\|\mathbf{I}-\mathbf{B H}_{\mathbf{I}}^{\mathbf{0}} \mathbf{A}\right\|^{2}+\mathrm{E}_{H}\left[\left\|\mathbf{B} \mathbf{T}_{\mathbf{I}} \mathbf{A}\right\|^{2}\right]+\operatorname{tr}\left[\mathbf{B} \mathbf{K}_{\mathbf{w}_{\mathbf{I}}} \mathbf{B}^{\mathrm{H}}\right]
\end{aligned}
$$

We note that

$$
\begin{aligned}
& \mathrm{E}_{H}\left[\left\|\mathbf{B} \mathbf{T}_{\mathbf{I}} \mathbf{A}\right\|^{2}\right]=\operatorname{tr}\left[\mathrm{E}_{H}\left[\mathbf{B} \mathbf{T}_{\mathbf{I}} \mathbf{A} \mathbf{A}^{\mathrm{H}} \mathbf{T}_{\mathbf{I}}{ }^{\mathrm{H}} \mathbf{B}^{\mathrm{H}}\right]\right] \\
& =\operatorname{tr}\left[\mathbf{A}^{\mathrm{H}} \mathbf{R}_{\mathbf{T}}^{\mathrm{T}} \mathbf{A}\right] \operatorname{tr}\left[\mathbf{B} \mathbf{R}_{\mathbf{I R}} \mathbf{B}^{\mathrm{H}}\right]
\end{aligned}
$$

where (14b) follows from [17, Ch.7]. We now have

$$
\begin{aligned}
\mathrm{E}_{H}[\varepsilon(\mathbf{A}, \mathbf{B})]= & \operatorname{tr}\left[\mathbf{A}^{\mathrm{H}} \mathbf{H}_{\mathbf{I}}^{\mathbf{0}^{\mathrm{H}}} \mathbf{B}^{\mathrm{H}} \mathbf{B} \mathbf{H}_{\mathbf{I}}^{\mathbf{0}} \mathbf{A}\right]-2 \operatorname{Re}\left[\operatorname{tr}\left[\mathbf{B} \mathbf{H}_{\mathbf{I}}^{\mathbf{0}} \mathbf{A}\right]\right]+n_{s} \\
& +\operatorname{tr}\left[\mathbf{A}^{\mathrm{H}} \mathbf{R}_{\mathbf{T}}^{\mathrm{T}} \mathbf{A}\right] \operatorname{tr}\left[\mathbf{B} \mathbf{R}_{\mathbf{I R}} \mathbf{B}^{\mathrm{H}}\right]+\operatorname{tr}\left[\mathbf{B} \mathbf{K}_{\mathbf{w}_{\mathbf{I}}} \mathbf{B}^{\mathrm{H}}\right]
\end{aligned}
$$

where $\operatorname{Re}[z]$ denotes the real part of $z \in \mathbb{C}$. Similarly the average energy harvested can be expressed as follows

$$
\begin{aligned}
\mathrm{E}_{H}[\mathcal{J}(\mathbf{A})] & =\mathrm{E}_{H}\left[\operatorname{tr}\left[\left(\mathbf{H}_{\mathbf{E}}^{\mathbf{0}}+\mathbf{T}_{\mathbf{E}}\right) \mathbf{A} \mathbf{A}^{\mathrm{H}}\left(\mathbf{H}_{\mathbf{E}}^{\mathbf{0}}+\mathbf{T}_{\mathbf{E}}\right)^{\mathrm{H}}\right]\right] \\
& =\operatorname{tr}\left[\mathbf{A}^{\mathrm{H}}\left(\mathbf{H}_{\mathbf{E}}^{\mathbf{0}}\right)^{\mathrm{H}} \mathbf{H}_{\mathbf{E}}^{\mathbf{0}} \mathbf{A}\right]+\operatorname{tr}\left[\mathbf{A}^{\mathrm{H}} \mathbf{R}_{\mathbf{T}}^{\mathrm{T}} \mathbf{A}\right] \operatorname{tr}\left[\mathbf{R}_{\mathbf{E R}}\right)(16)
\end{aligned}
$$

We now go back to the main problem considered in this section, the precoder design problem in (9). We observe that the objective function, i.e. (15), is a convex quadratic function in A. Similarly, the average energy harvested, (16), and the transmitter power constraints are also convex quadratic functions of $\mathbf{A}$. Nevertheless, the resulting 


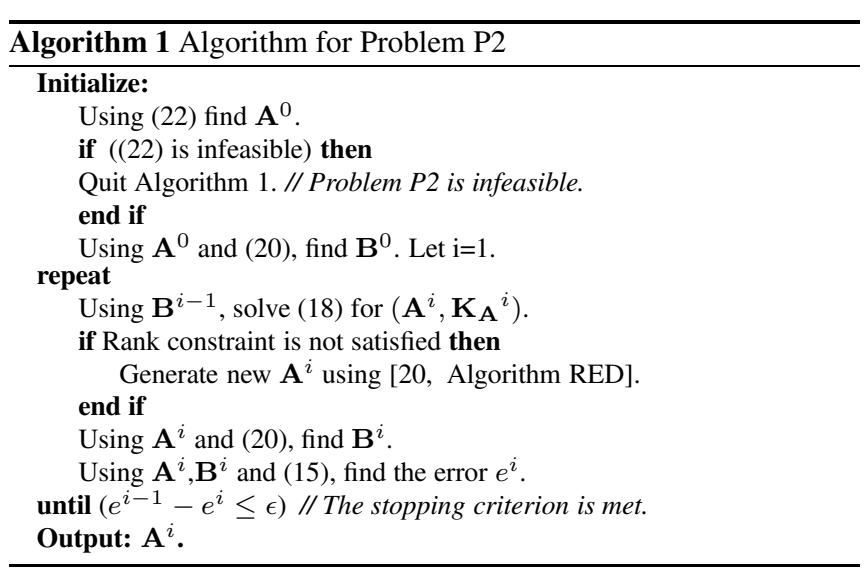

optimization problem is not convex since the energy harvesting constraint bounds a convex function from below, hence it does not form a convex constraint.

We now introduce a tight relaxation to the optimization problem in (9). We introduce a new variable $\mathbf{K}_{\mathbf{A}}=\mathbf{A} \mathbf{A}^{\mathbf{H}}$. Hence the objective function can be written as follows

$\bar{\varepsilon}_{K}\left(\mathbf{A}, \mathbf{K}_{\mathbf{A}}, \mathbf{B}\right)=\operatorname{tr}\left[\mathbf{H}_{\mathbf{I}}^{\mathbf{0}^{\mathrm{H}}} \mathbf{B}^{\mathrm{H}} \mathbf{B} \mathbf{H}_{\mathbf{I}}^{\mathbf{0}} \mathbf{K}_{\mathbf{A}}\right]-2 \operatorname{Re}\left[\operatorname{tr}\left[\mathbf{B} \mathbf{H}_{\mathbf{I}}^{\mathbf{0}} \mathbf{A}\right]\right]+n_{s}$ $+\operatorname{tr}\left[\mathbf{R}_{\mathbf{T}}^{\mathrm{T}} \mathbf{K}_{\mathbf{A}}\right] \operatorname{tr}\left[\mathbf{B} \mathbf{R}_{\mathbf{I R}} \mathbf{B}^{\mathrm{H}}\right]+\operatorname{tr}\left[\mathbf{B} \mathbf{K}_{\mathbf{w}_{\mathbf{I}}} \mathbf{B}^{\mathrm{H}}\right]$

The average energy harvested can be written as

$$
\overline{\mathcal{J}}_{K}\left(\mathbf{K}_{\mathbf{A}}\right)=\operatorname{tr}\left[\left(\mathbf{H}_{\mathbf{E}}^{\mathbf{0}}\right)^{\mathrm{H}} \mathbf{H}_{\mathbf{E}}^{\mathbf{0}} \mathbf{K}_{\mathbf{A}}\right]+\operatorname{tr}\left[\mathbf{R}_{\mathbf{T}}^{\mathrm{T}} \mathbf{K}_{\mathbf{A}}\right] \operatorname{tr}\left[\mathbf{R}_{\mathbf{E R}}\right]
$$

Hence the optimization problem in (9) can be equivalently expressed as

$$
\begin{array}{cl}
\min _{\mathbf{A}, \mathbf{K}_{\mathbf{A}}} & \bar{\varepsilon}_{K}\left(\mathbf{A}, \mathbf{K}_{\mathbf{A}}, \mathbf{B}\right) \\
\text { s.t. } & \overline{\mathcal{J}}_{K}\left(\mathbf{K}_{\mathbf{A}}\right) \geq \gamma, \\
& \operatorname{tr}\left[\mathbf{K}_{\mathbf{A}}\right] \leq P \\
& \mathbf{K}_{\mathbf{A}}=\mathbf{A A}^{\mathrm{H}} .
\end{array}
$$

Now the energy harvesting constraint is a linear function of the optimization variables. Nevertheless, the problem is not convex due to the equality constraint in (17d). We relax this constraint as $\mathbf{K}_{\mathbf{A}} \succeq$ $\mathbf{A A}^{\mathrm{H}}$. Hence the relaxed problem can be expressed as follows

$$
\begin{array}{cl}
\min _{\mathbf{A}, \mathbf{K}_{\mathbf{A}}} & \bar{\varepsilon}_{K}\left(\mathbf{A}, \mathbf{K}_{\mathbf{A}}, \mathbf{B}\right) \\
\text { s.t. } & (17 \mathrm{~b}),(17 \mathrm{c}), \\
& \mathbf{K}_{\mathbf{A}} \succeq \mathbf{A} \mathbf{A}^{\mathrm{H}} .
\end{array}
$$

This is a convex optimization problem, and it can be solved by standard numerical optimization tools, such as SDPT3 and CVX [18,19]. In the following result, we observe that this convex relaxation is tight:

Lemma 3.1 Let (18) be solvable. Then the optimum error values for (9) and the relaxed problem in (18) are equal and can be attained. An optimal solution for (9) can be constructed from an optimal solution of (18).

The proof is given in Sec. 7. In Sec. 4, we use this result as an intermediate step for solving the joint precoder-receiver filter design problem.

\section{JOINT PRECODER AND RECEIVER FILTER DESIGN}

In this section, we focus on Scenario II and discuss the precoder and receiver filter design problem in (10). We observe that, in general, (10) is not a convex optimization problem in $(\mathbf{A}, \mathbf{B})$. To see this, for instance, we recall that for fixed $\mathbf{B}$, the optimization problem is not convex as discussed in the previous section.
We now derive the optimum filter $\mathbf{B}$ for fixed precoder $\mathbf{A}$. We are interested in finding the filter $\mathbf{B}$ that minimizes the following error expression

$$
\begin{aligned}
\mathrm{E}_{H}[\varepsilon(\mathbf{A}, \mathbf{B})]= & \mathrm{E}_{H, S}\left[\left\|\mathbf{s}-\mathbf{B y}_{\mathbf{I}}\right\|^{2}\right] \\
(19) & \operatorname{tr}\left[\mathbf{B}\left(\mathbf{H}_{\mathbf{I}}^{\mathbf{0}} \mathbf{A} \mathbf{A}^{\mathrm{H}}\left(\mathbf{H}_{\mathbf{I}}^{\mathbf{0}}\right)^{\mathrm{H}}+\mathbf{K}_{\mathbf{w}_{\mathbf{I}}}\right) \mathbf{B}^{\mathrm{H}}\right]-2 \operatorname{Re}\left[\operatorname{tr}\left[\mathbf{B} \mathbf{H}_{\mathbf{I}}^{\mathbf{0}} \mathbf{A}\right]\right] \\
& +n_{s}+\operatorname{tr}\left[\mathbf{A}^{\mathrm{H}} \mathbf{R}_{\mathbf{T}}^{\mathrm{T}} \mathbf{A}\right] \operatorname{tr}\left[\mathbf{B} \mathbf{R}_{\mathbf{I R}} \mathbf{B}^{\mathrm{H}}\right] .
\end{aligned}
$$

By taking the derivative, and equating to zero, we find the optimal $\mathbf{B}$ as

$$
\mathbf{B}^{*}=\mathbf{A}^{\mathrm{H}}\left(\mathbf{H}_{\mathbf{I}}^{\mathbf{0}}\right)^{\mathrm{H}}\left(\mathbf{H}_{\mathbf{I}}^{\mathbf{0}} \mathbf{A} \mathbf{A}^{\mathrm{H}} \mathbf{H}_{\mathbf{I}}^{\mathbf{0}^{\mathrm{H}}}+\operatorname{tr}\left[\mathbf{A}^{\mathrm{H}} \mathbf{R}_{\mathbf{T}}^{\mathrm{T}} \mathbf{A}\right] \mathbf{R}_{\mathbf{I R}}+\mathbf{K}_{\mathbf{w}_{\mathbf{I}}}\right)^{-1} \text {. }
$$

Substituting $\mathbf{B}^{*}$ back into (19), the average mean-square error can be expressed as

$$
\mathrm{E}_{H}[\varepsilon(\mathbf{A}, \mathbf{B})]=\operatorname{tr}\left[\left(\mathbf{I}+\left(\mathbf{H}_{\mathbf{I}}^{\mathbf{0}} \mathbf{A}\right)^{\mathrm{H}} \overline{\mathbf{K}}_{\mathbf{w}_{\mathbf{I}}}^{-1} \mathbf{H}_{\mathbf{I}}^{\mathbf{0}} \mathbf{A}\right)^{-1}\right],
$$

where $\overline{\mathbf{K}}_{\mathbf{w}_{\mathbf{I}}}=\operatorname{tr}\left[\mathbf{A}^{\mathrm{H}} \mathbf{R}_{\mathbf{T}}^{\mathrm{T}} \mathbf{A}\right] \mathbf{R}_{\mathbf{I R}}+\mathbf{K}_{\mathbf{w}_{\mathbf{I}}}$. Hence the optimization problem in (10) can be equivalently written as the minimization of (21) over the precoder A subject to (3) and (8).

The error expression in (21) has a form similar to the error expression for the classical MMSE estimation where full CSI is available. Here, in the robust stochastic scenario, the noise covariance $\mathbf{K}_{\mathbf{w}_{\mathbf{I}}}$ is replaced by the modified expression $\overline{\mathbf{K}}_{\mathbf{w}_{\mathbf{I}}}$. We note that $\overline{\mathbf{K}}_{\mathrm{w}_{\mathbf{I}}}$ in general depends on $\mathbf{A}$, which makes this optimization problem very difficult to tackle.

To find a design for (10), we propose an alternating minimization approach. This method is summarized in Algorithm I. Here we take turns in fixing $\mathbf{A}$ and $\mathbf{B}$. For the fixed $\mathbf{B}$ step, by Lemma 3.1, an optimal solution for $\mathbf{A}$ can be found using the relaxation in (18). Details regarding how an optimal $\mathbf{A}$ is found from a solution of (18) is given in Sec. 7. For the fixed A step, an optimal B is found using (20). We initialize the algorithm by solving the following problem which maximizes the average energy harvested

$$
\max _{\mathbf{K}_{\mathbf{A}}} \overline{\mathcal{J}}_{K}\left(\mathbf{K}_{\mathbf{A}}\right)
$$

subject to (17b) and (17c), and using [20, Algorithm RED]. This alternating minimization technique is guaranteed to converge since in both fixed $\mathbf{A}$ and fixed $\mathbf{B}$ steps, the objective function decreases.

We note the model considered and subsequently the error expression studied in [11, eqn.18] is different from (21). The two expressions would be equal if $\mathbf{R}_{\mathbf{I R}}=\mathbf{I}$. In our formulation, this is not required. It is also worth mentioning that from a precoder design point of view, the analysis in [11] is restricted to the case where $n_{t} \leq n_{s}$, since it is not clear whether it is possible to produce a solution in the form $\mathbf{A} \in \mathbb{C}^{n_{t} \times n_{s}}$ satisfying $\mathbf{K}_{\mathbf{A}}=\mathbf{A} \mathbf{A}^{\mathrm{H}}$ from a transmit covariance matrix $\left(\mathbf{K}_{\mathbf{A}} \in \mathbb{C}^{n_{t} \times n_{t}}\right)$ found by solving [11, eqn.20] if $n_{t}>n_{s}$.

\section{NUMERICAL RESULTS}

For our numerical results, we consider the practical channel models considered in $[14,15]$. In particular the antenna correlation matrices are given by

$$
\left[\mathbf{R}_{c}\right]_{m, n}=e^{-j 2 \pi(n-m) d_{c} \cos \left(\theta_{c}^{0}\right)} e^{-0.5\left(2 \pi(n-m) d_{c} \sin \left(\theta_{c}\right) \sigma_{c}\right)^{2}},
$$

where $c=T, I R, E R$ corresponds to the transmitter, the information receiver and the energy receiver respectively. The normalized antenna spacing at the transmitter and the information/energy receiver are denoted by $d_{T}$ and $d_{I R} / d_{E R}$. Here $\theta_{T} \sim \mathcal{N}\left(\theta_{T}^{0}, \sigma_{T}^{2}\right)$ denotes the angle of departure for the transmitter. Similarly the angle of arrival for information/energy receiver is given by $\theta_{c} \sim \mathcal{N}\left(\theta_{c}^{0}, \sigma_{C}^{2}\right)$ where $c=I R / c=E R$. The mean channel component is given by

$$
\mathbf{H}_{c}^{0}=\sum_{1}^{L} \beta_{i} \mathbf{a}_{\mathbf{c}}\left(\theta_{c, i}\right) \mathbf{a}_{\mathbf{c}}^{\mathrm{T}}\left(\theta_{T, i}\right),
$$




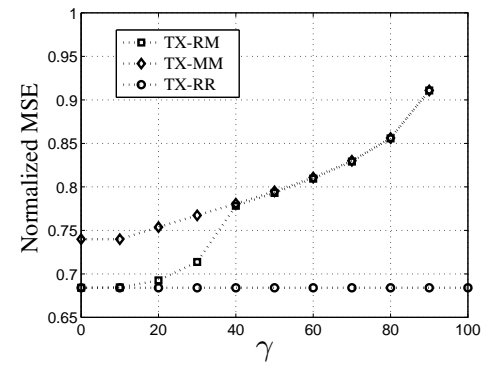

Fig. 1. Average mean-square error versus energy harvesting requirement, $K=0.1$.

where $c=T, I R, E R$. Here $\mathbf{a}_{\mathbf{T}}\left(\theta_{T, i}\right)$ is the array steering vector at the transmitter and $\mathbf{a} \mathbf{I R}\left(\theta_{I R, i}\right) / \mathbf{a}_{\mathbf{E R}}\left(\theta_{E R, i}\right)$ is the array response vector at IR/ER corresponding to $i$ th dominant path in the IR/ER channel. $\beta_{i}$ is the corresponding complex path amplitude. Here

$$
\mathbf{a}_{\mathbf{c}}(\theta)=\left[\begin{array}{lll}
1 & e^{j 2 \pi d_{c} \cos (\theta)} \ldots & e^{j 2 \pi\left(n_{c}-1\right) d_{c} \cos (\theta)}
\end{array}\right] .
$$

The Rician-K factors for information and energy receiver channels are given as follows: $K_{c}=\left\|\mathbf{H}_{c}^{0}\right\|^{2} / \mathrm{E}\left[\left\|\mathbf{T}_{c}\right\|^{2}\right]$, where $c=I, E$ [14]. Rician-K factor gives a parametrization for the ratio of the power in the mean component of the channel to the power in the fading component $[14,15]$. We consider the following parameters for our experiments: $d_{c}=0.5, \sigma_{c}^{2}=\pi / 128$ for $c=T, I R, E R$; $L=1, \beta_{1}=1, \theta_{I R, 1}=\theta_{I R}^{0}=\pi / 6, \theta_{E R, 1}=\theta_{E R}^{0}=\pi / 3$, $\theta_{T, 1}=\theta_{T}^{0}=\pi / 4 . \quad K_{I}=K_{E}=K \in\{0.1,1\}$. Let $n_{t}=$ $4, n_{r}=n_{e}=4, n_{s}=2, \mathbf{K}_{\mathbf{w}_{\mathbf{I}}}=\sigma_{w, I}^{2} \mathbf{I}, \sigma_{w, I}^{2}=1, P=1000(\mathrm{Ws})$. $\epsilon=10^{-6} n_{s}$. We assume that the system parameters, including the power constraint and the energy harvesting constraints, are scaled to the proper ranges. Discussions on the admissible values can be found in [1]. The associated convex optimization problems are solved using $[18,19]$.

In our numerical results we compare three different strategies: $T X-R R, T X-M M, T X-R M . T X-R R$ is the proposed design for the solution of (10) found by Algorithm 1. TX-MM corresponds to the strategy that uses the channel means as true channel matrices and optimizes the error under the energy harvesting constraint accordingly. This scenario corresponds to solving (10) with $\mathbf{R}_{\mathbf{T}}=\overline{\mathbf{0}}$, $\mathbf{R}_{\mathrm{IR}}=\overline{\mathbf{0}}, \mathbf{R}_{\mathrm{ER}}=\overline{\mathbf{0}}$ where $\overline{\mathbf{0}}$ is the zero matrix of appropriate dimensions. TX-RM corresponds to the robust error strategy found under a mean energy harvesting channel. Here transmitter assumes the channel mean is the actual channel realization in the energy harvesting channel whereas it provides a robust solution for the information transmission channel. This scenario corresponds to solving (10) with $\mathbf{R}_{\mathbf{E R}}=\overline{0}$. In our results, for all scenarios, the mean-square error (MSE) performance is reported as the average over the channel, i.e. evaluating (15) with the estimator in (20) for B. The MSE values normalized by dividing the MSE with $n_{s}=\operatorname{tr}\left[\mathbf{K}_{\mathbf{s}}\right]$ are presented in the figures.

We now study the trade-off between the average error and the energy harvesting requirements in Fig. 1 for $K=0.1$ and in Fig. 2 for $K=1$. We observe that the best error performance is exhibited by the fully robust solution $T X-R R$. It is observed that the advantage of the robust solution is more prominent for high levels of energy harvesting demand. For low energy harvesting requirements $T X-R R$ and $T X-R M$ show similar performance. In this range, the transmissions solely optimized for information transfer provide enough power to meet relatively low levels of energy harvesting requirements. For higher values of $\mathrm{EH}$ constraints, the main advantage of $T X-R R$ over $T X-R M$ is the fact that the transmitter is aware of the additional scat-

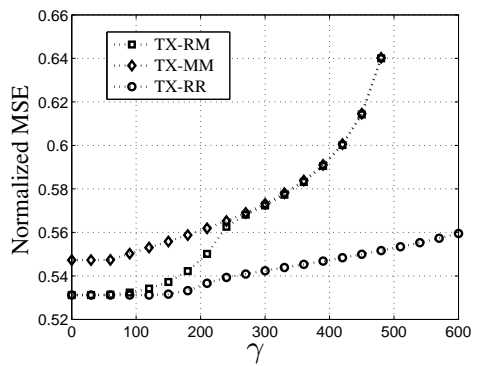

Fig. 2. Average mean-square error versus energy harvesting requirement, $K=1$.

tering and can adjust its transmission to take advantage of these to deliver power to the energy receiver. The fully non-robust solution (TX-MM) performs worst as expected. The close performance of $T X$ $R M$ and $T X-M M$ for high energy harvesting requirements is due to the necessity for the transmitter to optimize its transmission to meet these high requirements leaving little room for robust error reduction. Comparing Fig. 1 with Fig. 2 we observe that, as expected, the gap between the fully robust solution and the other solutions is smaller for the $K=1$ case, which is the case when the scattering component of the channel is less dominant.

\section{CONCLUSIONS}

Robust solutions for precoder design in SWIPT systems are investigated for MIMO channels with Rician fading. Our design methodologies allowed us to provide solutions even for scenarios where the number of transmit antennas is larger than the length of data vector. We have illustrated that significant gains compared to non-robust designs are obtained by the proposed robust designs, especially in cases where the scattering component of the channel is strong.

\section{APPENDIX}

Here we give the proof of Lemma 3.1. Using Schur complement [21, A.5.5], we equivalently write the expression in (18c) as a linear matrix inequality

$$
\mathbf{Z}_{\mathbf{A}}=\left[\begin{array}{cc}
\mathbf{I} & \mathbf{A}^{\mathrm{H}} \\
\mathbf{A} & \mathbf{K}_{\mathbf{A}}
\end{array}\right] \succeq 0
$$

We note that the optimization problem in (18) can be alternatively written in terms of the positive semi-definite variable $\mathbf{Z}_{\mathbf{A}}$ instead of $\mathbf{A}, \mathbf{K}_{\mathrm{A}}$.

We observe that (18) is the SDP relaxation of (9). (One can, for instance, see [20, 2.7] for the general form of the SDP relaxations of such quadratically constrained optimization problems.) By [20, Thm 2.2], (9) and its SDP relaxation have the same optimal value if the relaxation is solvable and the number of constraints in the original problem (9) is equal to or smaller than $2 n_{s}$. We also observe that the dual of (18) is strictly feasible since the regularity condition in [20, 2.10] holds. (This is due to the fact that the matrix associated with the power constraints, identity, is positive definite.) Hence together with the feasibility of (9), this implies solvability of SDP relaxation [20, Cor. 2.1].

The above argument shows that the two problems have the same optimum value. With regard to the optimal solutions, we observe the following: An optimal solution for (9) can be constructed from a solution of (18) using [20, Algorithm RED] or equivalently [22, Algorithm 1]. Using these algorithms on an optimal $\mathbf{Z}_{\mathbf{A}}$ will result in a rank-constrained optimal $\mathbf{Z}_{\mathbf{A}}$ solution. Due to [20, Lemma 2.1], an optimal solution $\mathbf{A}$ for (9) is given by the lower left $n_{t} \times n_{s}$ matrix of this rank-constrained $\mathbf{Z}_{\mathrm{A}}$. 


\section{REFERENCES}

[1] R. Zhang and C. K. Ho, "MIMO broadcasting for simultaneous wireless information and power transfer," IEEE Transactions on Wireless Communications, vol. 12, pp. 1989-2001, May 2013.

[2] J. Xu, L. Liu, and R. Zhang, "Multiuser MISO beamforming for simultaneous wireless information and power transfer," IEEE Transactions on Signal Processing, vol. 62, pp. 47984810, Sept 2014.

[3] L. Liu, R. Zhang, and K.-C. Chua, "Secrecy wireless information and power transfer with MISO beamforming," IEEE Transactions on Signal Processing, vol. 62, pp. 1850-1863, April 2014.

[4] H. Sampath, P. Stoica, and A. Paulraj, "Generalized linear precoder and decoder design for MIMO channels using the weighted MMSE criterion," IEEE Transactions on Communications, vol. 49, no. 12, pp. 2198-2206, 2001.

[5] M. Rupf, F. Tarkoy, and J. Massey, "User-separating demodulation for code-division multiple-access systems," IEEE Journal on Selected Areas in Communications, vol. 12, pp. 786-795, Jun 1994.

[6] H. Shen, J. Wang, W. Xu, Y. Rong, and C. Zhao, "A worst-case robust MMSE transceiver design for nonregenerative MIMO relaying," IEEE Transactions on Wireless Communications, vol. 13, pp. 695-709, February 2014.

[7] H. Zhang, K. Song, Y. Huang, and L. Yang, "Energy harvesting balancing technique for robust beamforming in multiuser MISO SWIPT system," in 2013 International Conference on Wireless Communications and Signal Processing, pp. 1-5, Oct 2013.

[8] D. Li, C. Shen, and Z. Qiu, "Two-way relay beamforming for sum-rate maximization and energy harvesting," in 2013 IEEE International Conference on Communications (ICC), pp. 3115-3120, June 2013.

[9] D. Ng, E. Lo, and R. Schober, "Robust beamforming for secure communication in systems with wireless information and power transfer," IEEE Transactions on Wireless Communications, vol. 13, pp. 4599-4615, Aug 2014.

[10] Q. Li, W.-K. Ma, and A.-C. So, "Robust artificial noise-aided transmit optimization for achieving secrecy and energy harvesting," in 2014 IEEE International Conference on Acoustics, Speech and Signal Processing (ICASSP), pp. 1596-1600, May 2014.

[11] C. Xing, N. Wang, J. Ni, Z. Fei, and J. Kuang, "MIMO beamforming designs with partial CSI under energy harvesting constraints," IEEE Signal Processing Letters, vol. 20, pp. 363-366, April 2013.

[12] S. Wu, Q. Li, W.-K. Ma, and A.-C. So, "Robust transmit designs for an energy harvesting multicast system," in 2014 IEEE International Conference on Acoustics, Speech and Signal Processing (ICASSP), pp. 4748-4752, May 2014.

[13] C.-N. Chuah, D. Tse, J. Kahn, and R. Valenzuela, "Capacity scaling in MIMO wireless systems under correlated fading," IEEE Transactions on Information Theory, vol. 48, pp. 637650, Mar 2002.
[14] H. Bolcskei, M. Borgmann, and A. Paulraj, "Impact of the propagation environment on the performance of spacefrequency coded MIMO-OFDM," IEEE Journal on Selected Areas in Communications, vol. 21, pp. 427-439, Apr 2003.

[15] M. McKay and I. Collings, "General capacity bounds for spatially correlated Rician MIMO channels," IEEE Transactions on Information Theory, vol. 51, pp. 3121-3145, Sept 2005.

[16] J. Wang and D. Palomar, "Robust MMSE precoding in MIMO channels with pre-fixed receivers," IEEE Transactions on Signal Processing, vol. 58, pp. 5802-5818, Nov 2010.

[17] A. Gupta and D. Nagar, Matrix Variate Distributions. Chapmann \& Hall, 2000.

[18] R. H. Tütüncü, K. C. Toh, and M. J. Todd, "Solving semidefinite-quadratic-linear programs using SDPT3," Mathematical Programming, vol. 95, no. 2, pp. 189-217, 2003.

[19] CVX Research Inc., "CVX: Matlab software for disciplined convex programming 2.0." http://cvxr.com/cvx, 2012.

[20] A. Beck, "Convexity properties associated with nonconvex quadratic matrix functions and applications to quadratic programming," Journal of Optimization Theory and Applications, vol. 142, no. 1, pp. 1-29, 2009.

[21] S. Boyd and L. Vandenberghe, Convex Optimization. Cambridge University Press, 2004.

[22] Y. Huang and D. Palomar, "Rank-constrained separable semidefinite programming with applications to optimal beamforming," IEEE Transactions on Signal Processing, vol. 58, no. 2, pp. 664-678, 2010. 